\title{
Multi-User MIMO-OFDM for Indoor Visible Light Communication Systems
}

\author{
Qi Wang ${ }^{\dagger}$, Zhaocheng Wang ${ }^{\dagger}$, Chen Qian ${ }^{\dagger}$, Jinguo Quan ${ }^{\ddagger}$, and Linglong Dai ${ }^{\dagger}$ \\ ${ }^{\dagger}$ Tsinghua National Laboratory for Information Science and Technology (TNList), \\ Department of Electronic Engineering, Tsinghua University, Beijing 100084, China \\ ${ }^{\ddagger}$ Division of Information Science \& Technology, Shenzhen Graduate School, \\ Tsinghua University, Shenzhen 518055, China
}

\begin{abstract}
In this paper, we investigate an indoor multiuser visible light communication system employing multipleinput multiple-output (MIMO) orthogonal frequency-division multiplexing (OFDM). For each subcarrier in OFDM, the corresponding precoding matrix is calculated in the frequency domain to eliminate multi-user interference. The distances of the multiple transmitter-receiver links are different, which results in various temporal delays and phase differences in the frequency domain. Phase information is firstly considered, whereby complex instead of real channel matrices are used for precoding, which reduces the channel correlation and achieves better performance. Moreover, two DC bias and scaling factor calculation schemes are proposed, and their performances are compared with zero forcing and minimum mean-squared error (MMSE) precoding techniques.

Index Terms-Visible light communication, multiple-input multiple-output, orthogonal frequency-division multiplexing, multi-user, precoding.
\end{abstract}

\section{INTRODUCTION}

Visible light communication (VLC) has recently attracted great attention due to its distinct features such as wide unregulated bandwidth, low cost and high security [1]-[3]. It has been considered as a promising supplementary for traditional radio frequency communications, and will play an important role in $5 \mathrm{G}$ and beyond wireless communications, especially in indoor applications [4]-[6]. In indoor VLC systems, lightemitting diodes (LEDs) are used for data transmission, which are also increasingly deployed for illumination and display. Intensity modulation with direct detection (IM/DD) is applied in VLC for low cost implementation, where the information is conveyed through the intensity of LEDs and detected by a photodiode (PD) at the receiver.

In order to achieve sufficient illumination, multiple LED units are usually installed in a single room, where multipleinput multiple-output (MIMO) techniques can be naturally employed in indoor VLC systems. Recently, multi-user MIMO (MU-MIMO) has been studied for VLC, and several precoding

This work was supported by National Key Basic Research Program of China (Grant No. 2013CB329203), National Nature Science Foundation of China (Grant No. 61271266), Shenzhen Visible Light Communication System Key Laboratory (ZDSYS20140512114229398), Shenzhen Wireless over Visible Light Communication Technology Engineering Laboratory(SDRC[2012]No.1440) and Shenzhen Peacock Plan (No. 1108170036003286) schemes such as zero forcing, minimum mean-squared error (MMSE), and block diagonalization have been proposed [7][9]. However, indoor VLC channels are typically highly correlated when line-of-sight (LOS) scenarios are considered, which is unfavorable for MIMO techniques and leads to the degraded performance [10], [11]. As a spectrally efficient modulation scheme, orthogonal frequency-division multiplexing (OFDM) is intensively employed in VLC and up to several Gbps pointto-point transmissions have been reported [12]-[16]. MIMOOFDM is widely used in radio frequency (RF) systems to support multi-user service and provide high data rate transmission [17], [18], but it has rarely been studied in VLC systems. A MIMO-OFDM VLC system has been demonstrated in [19], but an imaging diversity receiver is required to achieve parallel transmission, which is infeasible for multi-user scenarios.

In this paper, multi-user MIMO-OFDM (MU-MIMOOFDM) is investigated for indoor VLC systems. For each subcarrier in OFDM, the corresponding precoding matrix is calculated in the frequency domain to eliminate multi-user interference. Two different DC bias and scaling schemes are considered for multiple LEDs to obtain non-negative signals. Since the distances of the multiple transmitter-receiver links are different, their temporal delays are also different, resulting in phase differences in the frequency domain. The phase difference can not be neglected when wide-band systems are used, especially for the subcarriers with high indexes. Different from state-of-the-art schemes, complex rather than real channel matrices are used for precoding, which reduces the channel correlation and achieves better performance.

The following notations are used throughout this paper. Uppercase letters denote frequency-domain signals, e.g., $X$, whereas the corresponding lowercase letters represent timedomain signals, e.g., $x$. Boldface letters denote vectors or matrices, e.g., $\mathbf{x}$ or $\mathbf{X} .(\cdot)^{*}$ is the conjugate operator. $(\cdot)^{T}$ and $(\cdot)^{H}$ are the transpose and Hermitian transpose operators for vector or matrix. $(\cdot)^{\dagger}$ and $(\cdot)^{-1}$ are pseudo-inverse and inverse operators for a matrix, respectively. $E(\cdot)$ denotes the expectation operation.

\section{SySTEM MODEL}

A typical indoor VLC system is shown in Fig. 1. Multiple LED units are installed in a single room for illumination and 


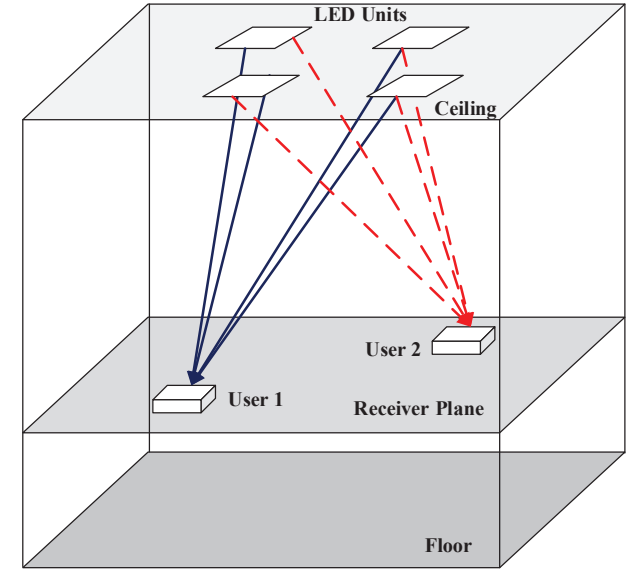

Fig. 1. Illustration of indoor multi-user VLC system.

cooperate to transmit information for multiple users at the same time. In this paper, we consider $N_{t}$ LED units and $N_{r}$ users each with a single PD, where we have $N_{t} \geq N_{r}$. The transmitted $N_{r} \times 1$ data vector $\mathbf{d}$ is firstly precoded into an $N_{t} \times 1$ transmitted vector $\mathbf{x}$. Since intensity modulation is used at the transmitter, the transmitted vector has to be real-valued and non-negative, and DC bias is required for each LED unit.

The DC channel gain from the $q$ th LED unit to the $p$ th user is given by [10]

$$
h_{p, q}^{D C}= \begin{cases}\frac{\rho_{p} A_{p}}{d_{p, q}^{2}} R\left(\phi_{q}\right) \cos \left(\varphi_{p, q}\right), & \varphi_{p, q} \leq \Psi_{c, p}, \\ 0, & \varphi_{p, q}>\Psi_{c, p},\end{cases}
$$

where $\rho_{p}$ is the PD responsivity coefficient, $d_{p, q}$ is the distance between the $q$ th LED unit and the $p$ th user, $\phi_{q}$ is the emission angle of $q$ th LED unit, $\varphi_{p, q}$ is the incidence angle of the light, and $\Psi_{c, p}$ is the receiver field of view (FOV) of the $p$ th user. The channel matrix $\left\{h_{p, q}^{D C}\right\}_{N_{r} \times N_{t}}$ is real-valued and usually highly correlated when the users are close. $A_{p}$ is the receiver collection area of the $p$ th user and it is calculated as

$$
A_{p}=\gamma^{2} A_{P D, p} / \sin \left(\Psi_{c, p}\right),
$$

where $\gamma$ denotes the concentrator refractive index of the PD, $A_{P D, p}$ is the PD area of the $p$ th user. In Eq. (1), $R\left(\phi_{p, q}\right)$ is the Lambertian radiant intensity given by [10]

$$
R\left(\phi_{q}\right)=\left((m+1) \cos ^{m}\left(\phi_{q}\right)\right) / 2 \pi,
$$

where $m$ is the order of Lambertian emission.

At the receiver, the optical signal is directly detected by the corresponding PD, which generates an electric signal proportional to the optical power. Besides that, shot noise and thermal noise are induced at the receiver, which can be modeled as real-valued additive white Gaussian noise (AWGN) with zero mean, and its variance is given by [10]

$$
\sigma_{p}^{2}=2 e P_{p} B+2 e \rho_{p} \chi_{a m b} A_{p}\left(1-\cos \left(\theta_{c, p}\right)\right) B+i_{a m p}^{2},
$$

where $e$ is the electronic charge, $\chi_{a m b}$ is the ambient light photocurrent, $B$ is the receiver bandwidth, and $i_{a m p}$ is the preamplifier noise current density. $P_{p}$ is the average received optical power at the $p$ th user collected from all the LED units.

\section{MU-MIMO-OFDM FOR VLC}

In MU-MIMO-OFDM system with $N$ subcarriers, the transmitted bit stream for the $p$ th user is firstly mapped onto the complex-valued symbols $D_{p, k}, k=0,1, \cdots, N-1$, according to the chosen constellations, such as quadrature amplitude modulation (QAM) or phase shift keying (PSK). Since real-valued output is required for intensity modulation, OFDM subcarriers should satisfy the Hermitian symmetry that $D_{p, k}=D_{p, N-k}^{*}, k=1,2, \cdots, N / 2-1$, and $D_{p, 0}$ and $D_{p, N / 2}$ are set to zero.

For each subcarrier, precoding is required at the transmitter to eliminate multi-user interference. Here we denote the precoding weights for the $k$ th $(k=0,1, \cdots, N-1)$ subcarrier as $\left\{W_{p, q, k}, 1 \leq p \leq N_{r}, 1 \leq q \leq N_{t}\right\}$. The precoding weights can be complex-valued since Hermitian symmetry constraint is imposed on the subcarriers to guarantee the time-domain signals real-valued. By adding up all the weighted symbols from $N_{r}$ users at the $q$ th LED unit, the frequency-domain signal can be written as

$$
X_{q, k}=\sum_{p=1}^{N_{r}} W_{p, q, k} D_{p, k} .
$$

After that, the frequency-domain signals are converted to the time domain by inverse fast Fourier transform (IFFT) as

$$
x_{q, n}=\frac{1}{\sqrt{N}} \sum_{k=0}^{N-1} X_{q, n} \exp \left(j \frac{2 \pi}{N} n k\right), n=0,1, \cdots, N-1 .
$$

At the beginning of each OFDM symbol $\mathbf{x}_{q}=$ $\left[x_{q, 0}, x_{q, 1}, \cdots, x_{q, N-1}\right]^{T}$, a cyclic prefix (CP) is added to eliminate the inter-symbol interference at the receiver. Since $x_{q, n}$ is a bipolar signal, a DC bias $P_{\mathrm{DC}, q}$ is added to the $q$ th transmitter to obtain non-negative signals for emission.

In existing MU-MIMO VLC systems, single-carrier modulations are used with limited bandwidth. Therefore, precoding is conducted in the time domain and only the DC channel gain is considered [7]-[9]. However, in order to achieve up to $100 \mathrm{Gbps}$ high data rate transmission [20], [21], wide bandwidth optical components are used. Since the distances of the multiple transmitter-receiver links are different, their temporal delays are also different, which can not be neglected in wide bandwidth scenarios. Therefore, the time-domain channel response from the $q$ th LED unit to the $p$ th user in (1) can be rewritten as

$$
h_{p, q}(t)=h_{p, q}^{D C} \delta\left(t-\frac{d_{p, q}}{c}\right),
$$

where $\delta(\cdot)$ denotes the Dirac delta function and $c$ is the speed of light. Correspondingly, the frequency-domain channel response for the $k$ th subcarrier is given by

$$
H_{p, q, k}=h_{p, q}^{D C} \exp \left(-\frac{j 2 \pi k B d_{p, q}}{N c}\right),
$$


where $j$ denotes the imaginary unit and $j=\sqrt{-1}$. It can be seen that when the temporal delay is considered, the frequency-domain channel response is complex-valued, which provides an extra dimension and reduces the channel correlation with the phase differences of multiple links.

\section{A. Precoding Design}

For the $p$ th user, the received signal can be written in the frequency domain after FFT as

$$
\begin{aligned}
R_{p, k} & =\sum_{q=1}^{N_{t}} H_{p, q, k} X_{q, k}+Z_{p, k} \\
& =\mathbf{H}_{p, k}^{T} \mathbf{W}_{p, k} D_{p, k}+\sum_{l \neq p}^{N_{t}} \mathbf{H}_{l, k}^{T} \mathbf{W}_{l, k} D_{l, k}+Z_{p, k},
\end{aligned}
$$

where $\mathbf{H}_{p, k}^{T}, \mathbf{W}_{p, k}$, and $\mathbf{W}_{l, k}$ are $N_{t} \times 1$ vectors, $Z_{p, k}$ denotes the equivalent noise on the $k$ th subcarrier. The first term $\mathbf{H}_{p, k}^{T} \mathbf{W}_{p, k} D_{p, k}$ in (9) is the useful signal for the $p$ th user, while the second term $\sum_{l \neq p}^{N_{t}} \mathbf{H}_{l, k}^{T} \mathbf{W}_{l, k} D_{l, k}$ is the inter-user interference.

Eq. (9) can be rewritten in the matrix form as

$$
\mathbf{R}_{k}=\mathbf{H}_{k} \mathbf{W}_{k} \mathbf{D}_{k}+\mathbf{Z}_{k},
$$

where $\mathbf{D}_{k}$ and $\mathbf{R}_{k}$ denote the transmitted and received symbol vectors, $\mathbf{H}_{k}=\left[\mathbf{H}_{1, k}, \mathbf{H}_{2, k}, \cdots, \mathbf{H}_{N_{r}, k}\right]^{T}$ and $\mathbf{W}_{k}=$ $\left[\mathbf{W}_{1, k} \mathbf{W}_{2, k} \cdots \mathbf{W}_{N_{r}, k}\right]$ represent the channel and precoding matrices.

Since the channel matrices for different subcarriers are different according to (8), their corresponding precoding matrices should be calculated separately. Several precoding schemes have been proposed for MU-MIMO in RF systems [22], [23]. In particular, we use two well-known techniques to eliminate the inter-user interference, namely, zero forcing and MMSE algorithms.

For zero forcing, the interference is eliminated by directly forcing the interference terms to be zeros, i.e., the matrix $\mathbf{H}_{k} \mathbf{W}_{k}$ is forced to be diagonal as [7], [22]

$$
\mathbf{H}_{k} \mathbf{W}_{k}=\operatorname{diag}\left(\boldsymbol{\lambda}_{k}\right) \text {, }
$$

where all the elements in $\boldsymbol{\lambda}_{k}$ are positive and the precoding matrix can be calculated by

$$
\mathbf{W}_{k}=\mathbf{H}_{k}^{\dagger} \operatorname{diag}\left(\boldsymbol{\lambda}_{k}\right)=\mathbf{H}_{k}^{H}\left(\mathbf{H}_{k} \mathbf{H}_{k}^{H}\right)^{-1} \operatorname{diag}\left(\boldsymbol{\lambda}_{k}\right) .
$$

When the channel matrix is ill-conditioned, zero forcing requires a large normalization factor, which will dramatically reduce the received power [23]. Therefore, when the signalto-noise ratio (SNR) at the receiver is low, noise instead of interference is dominant, which does not perform well.

In linear MMSE, however, a tradeoff between interference and noise is achieved based on which one is the dominant part in signal-to-interference-plus-noise ratio (SINR), and the precoding matrix is given by [23]

$$
\mathbf{W}_{k}=\mathbf{H}_{k}^{H}\left(\mathbf{H}_{k} \mathbf{H}_{k}^{H}+\operatorname{diag}\left(\boldsymbol{\sigma}_{\mathbf{Z}_{k}}^{2}\right)\right)^{-1} \operatorname{diag}\left(\boldsymbol{\lambda}_{k}\right),
$$

where $\boldsymbol{\sigma}_{\mathbf{Z}_{k}}^{2}$ denotes the variance vector of $\mathbf{Z}_{k}$.

\section{B. DC Bias and Scaling Factor}

The OFDM time-domain signal $x_{q, n}$ in (6) is bipolar, a DC bias $P_{\mathrm{DC}, q}$ is added to the $q$ th transmitter to obtain nonnegative signals for emission. According to the central limit theorem, $x_{q, n}$ approximates a Gaussian distribution when $N \geq$ 64 , and it might be very large [13]. Therefore, a DC bias can not necessarily make sure all the signals are non-negative and these signals should be clipped, leading to undesired clipping distortion. The DC bias is written as [24]

$$
P_{\mathrm{DC}, q}=\eta \sqrt{E\left\{x_{q, n}^{2}\right\}}
$$

which is usually given in the form of $10 \log _{10}\left(\eta^{2}+1\right) \mathrm{dB}$ since it represents the increase in electric power of original OFDM signals, where $\eta$ denotes the DC bias ratio. When DC bias is large, the clipping distortion would be very small. However, it is inefficient in terms of power since DC bias does not carry information. There is a tradeoff between the DC bias and clipping distortion and we can define a minimum DC bias ratio $\eta_{0}$ to avoid clipping distortion [24].

When precoding is applied at the transmitter, the electric power of the $N_{t}$ transmitters is different, which requires different minimum DC bias. If we use the minimum DC bias for each LED unit, we have

$$
P_{\mathrm{DC}, q}=\eta_{0} \sqrt{E\left\{x_{q, n}^{2}\right\}}, q=0,1, \cdots, N_{t}-1,
$$

and the emitted optical power of the $q$ th LED unit is given by

$$
P_{\text {opt }, q}=E\left\{x_{q, n}+P_{\mathrm{DC}, q}\right\}=E\left\{x_{q, n}\right\}+P_{\mathrm{DC}, q}=P_{\mathrm{DC}, q},
$$

where the equality holds since the expectation of $x_{q, n}$ is zero according to (6).

When the average optical power of all the LED units is given as $P$ for illumination requirement, a scaling factor should be added to the biased signal and the transmitted signal for the $q$ th LED unit is given by

$$
y_{q, n}=\alpha\left(x_{q, n}+P_{\mathrm{DC}, q}\right),
$$

where the scaling factor can be calculate as

$$
\alpha=\frac{N_{t} P}{\sum_{q=1}^{N_{t}} P_{\mathrm{DC}, q}}=\frac{N_{t} P}{\eta_{0} \sum_{q=1}^{N_{t}} \sqrt{E\left\{x_{q, n}^{2}\right\}}} .
$$

It can be seen in (16) that the emitted optical power in the $N_{t}$ LED units is different when the precoding matrix changes, which will affect the illumination performance of LEDs. In order to provide high quality illumination at the same time, we consider a unified DC bias scheme, where all the LED units use the same DC bias, i.e., the maximum DC bias for all the $N_{t}$ units to avoid clipping distortion, we have

$$
P_{\mathrm{DC}, q}=\eta_{0} \sqrt{\max _{1 \leqslant q \leqslant N_{t}}\left(E\left\{x_{q, n}^{2}\right\}\right)},
$$

and the corresponding scaling factor is given by

$$
\alpha=\frac{N_{t} P}{\sum_{q=1}^{N_{t}} P_{\mathrm{DC}, q}}=\frac{P}{\eta_{0} \sqrt{\max _{1 \leqslant q \leqslant N_{t}}\left(E\left\{x_{q, n}^{2}\right\}\right)}} .
$$


TABLE I

SIMULATION PARAMETERS FOR VLC SYSTEM CONFIGURATION

\begin{tabular}{|c|c|}
\hline Room size (length $\times$ width $\times$ height) & $5 \mathrm{~m} \times 5 \mathrm{~m} \times 3 \mathrm{~m}$ \\
\hline LED 1 coordinate & {$\left[\begin{array}{lll}1.25 & 1.25 & 3\end{array}\right]$} \\
\hline LED 2 coordinate & {$\left[\begin{array}{llll}1.25 & 3.75 & 3\end{array}\right]$} \\
\hline LED 3 coordinate & {$\left[\begin{array}{lll}3.75 & 1.25 & 3\end{array}\right]$} \\
\hline LED 4 coordinate & {$\left[\begin{array}{lll}3.75 & 3.75 & 3\end{array}\right]$} \\
\hline LED emission angle $\phi_{q}$ & $60 \mathrm{deg}$ \\
\hline $\mathrm{PD}$ area $A_{P D, p}$ & $1 \mathrm{~cm}^{2}$ \\
\hline PD responsivity coefficient $\rho_{p}$ & $0.4 \mathrm{~A} / \mathrm{W}$ \\
\hline PD concentrator refractive index $\gamma$ & 1.5 \\
\hline Lambertian emission mode number $m$ & 1 \\
\hline Receiver FOV angle $\Psi_{c, p}$ & $62 \mathrm{deg}$ \\
\hline Pre-amplifier noise density $i_{a m p}$ & $5 \mathrm{pA} / \mathrm{Hz}^{-1 / 2}$ \\
\hline Ambient light photocurrent $\chi_{a m p}$ & $10.93 \mathrm{~A} / \mathrm{m}^{2} / \mathrm{Sr}$ \\
\hline System bandwidth $B$ & $1 \mathrm{GHz}$ \\
\hline OFDM subcarrier number $N$ & 64 \\
\hline Cyclic prefix length $N_{C P}$ & 3 \\
\hline
\end{tabular}

\section{Simulation Results}

The performance of the MU-MIMO-OFDM is evaluated via simulations in a system with 4 LED units and 2 user$\mathrm{s}$ in terms of the aggregate achievable spectral efficiency, which is calculated with all the SINRs at the receiver by $\sum_{p=1}^{N_{r}} \log _{2}\left(1+\operatorname{SINR}_{p}\right)$. The simulation parameters are listed in Table I. The parameters for LEDs and PDs are chosen from [10]. Two cases of users' locations are considered. In Case 1, the user 1's coordinate is [ 2.52 .50 .85$]$, which is in the center of the room, while user 2's coordinate is [3.2 3.9 0.85]. It is a relatively good scenario since the two users are separated far enough, thus having a uncorrelated channel matrix. In Case 2, the two users' coordinates are [2.05 1.6 0.85] and [2.05 1.4 $0.85]$, which are very close and their corresponding channel matrix is ill-conditioned. Zero forcing and MMSE precoding schemes are used for both cases. The minimum DC bias factor $\eta_{0}$ is set to 3 so that less than $0.15 \%$ signals would be clipped at the transmitter and the clipping distortion is negligible.

Figure 2 depicts the spectral efficiency of each subcarrier in OFDM with the average emitted optical power $P=0 \mathrm{~dB}$. Since Hermitian symmetry is applied, only the 1st to 31 th subcarriers carry useful information. It can be seen that when the subcarrier index increases, the spectral efficiency improves, especially in Case 2. This is because the subcarrier with higher index has more phase difference, which makes the channel matrix more uncorrelated, and this improvement is more useful for correlated scenario as in Case 2.

Figure 3 shows the average spectral efficiency with different average emitted optical power. It can be seen that MMSE outperforms zero forcing in both cases. MMSE achieves more spectral efficiency when the optical power is low and the noise is the dominant part of SINR. The performance gain of MMSE becomes larger in Case 2 since its channel matrix is more illconditioned. Besides that, the system with minimum DC bias according to (15) and (18) achieves more spectral efficiency than that with unified DC bias according to (19) and (20) since more power are used for data transmission. However,

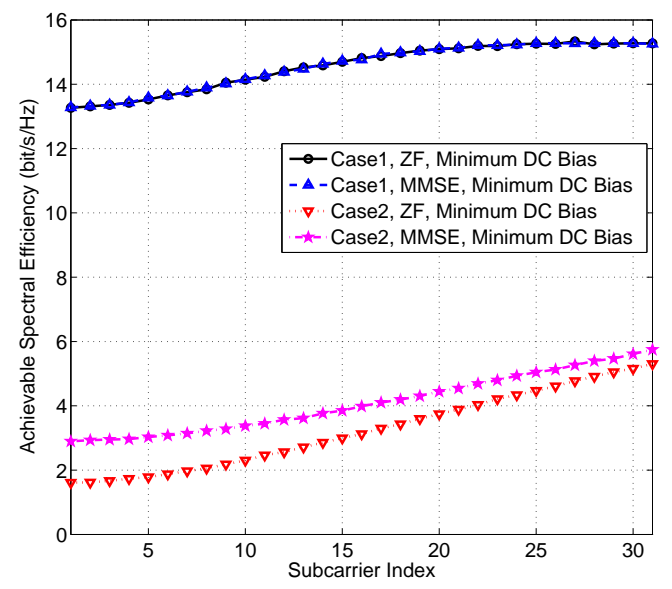

Fig. 2. Spectral efficiency of each subcarrier in OFDM with the average emitted optical power $P=0 \mathrm{~dB}$.

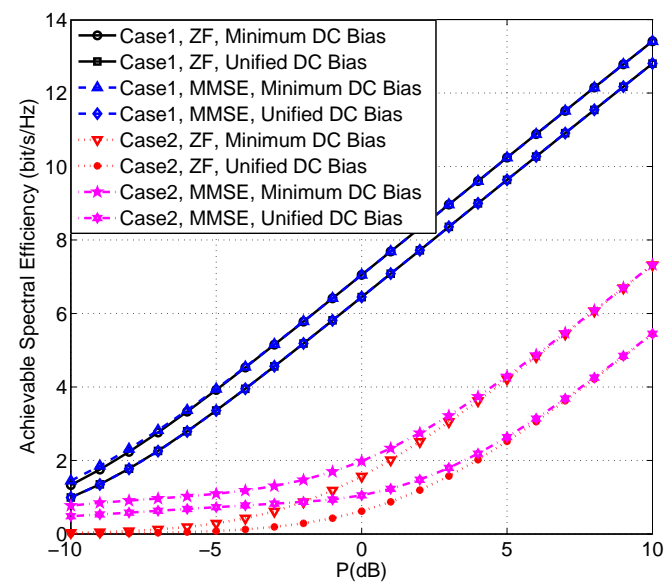

Fig. 3. Average spectral efficiency with different average emitted optical power.

the system with unified DC bias provides better illumination performance.

\section{CONCLUSION}

In this paper, MU-MIMO-OFDM is studied for indoor VLC systems. The distance differences of the multiple transmitterreceiver links are considered, resulting in phase differences in the frequency domain. Different from state-of-the-art schemes, zero forcing and MMSE precoding techniques are performed on the complex channel matrices for each subcarrier, which are less correlated when the phase differences are considered. Two DC bias and scaling schemes are proposed for different sceneries. Simulation results show that the subcarrier with higher index achieves more spectral efficiency, especially when the users are highly correlated. MMSE outperforms zero forcing when the optical power is low and more performance gain is achieved when the users are closer. 


\section{REFERENCES}

[1] R. Zhang, J. Wang, Z. Wang, Z. Xu, C. Zhao, and L. Hanzo, "Visible light communications in heterogeneous networks: pave the way for usercentric design," IEEE Wireless Communications, vol. 22, no. 2, pp. 8-16, Apr. 2015.

[2] Q. Gao, C. Gong, S. Li, and Z. Xu, "DD-informative modulation for visible light communications under lighting constraints," IEEE Wireless Communications, vol. 22, no. 2, pp. 54-60, Apr. 2015.

[3] Q. Wang, Z. Wang, S. Chen, and L. Hanzo, "Enhancing the decoding performance of optical wireless communication systems using receiverside predistortion," Optics Express, vol. 21, no. 25, pp. 30295-30305, Dec. 2013.

[4] S. Wu, H. Wang, and C. Youn, "Visible light communications for 5G wireless networking systems: from fixed to mobile communications," IEEE Network, vol. 28, no. 6, pp. 41-45, Nov.-Dec. 2014.

[5] Q. Wang, Q. Xie, Z. Wang, S. Chen, and L. Hanzo, "A universal lowcomplexity symbol-to-bit soft demapper," IEEE Trans. Veh. Technol., vol. 63, no. 1, pp. 119-130, Jan. 2014.

[6] J. Zhu, X. Lin, R. S. Blum and Y. Gu, "Parameter estimation from quantized observations in multiplicative noise environments," IEEE Transactions on Signal Processing, vol. 63, no. 15, pp. 4037-4050, Aug. 2015.

[7] Z. Yu, R. J. Baxley, and G. T. Zhou, "Multi-user MISO broadcasting for indoor visible light communication," in Proc. IEEE International Conference on Acoustics, Speech and Signal Processing (ICASSP), Vancouver, Canada, May 2013, pp. 4849-4853.

[8] H. Ma, L. Lampe, and S. Hranilovic, "Robust MMSE linear precoding for visible light communication broadcasting systems," in Proc. IEEE Globecom Workshops, Atlanta, GA, Dec. 2013, pp. 1081-1086.

[9] Y. Hong, J. Chen, Z. Wang, and C. Yu, "Performance of a precoding MIMO system for decentralized multiuser indoor visible light communications," IEEE Photonics Journal, vol. 5, no. 4, pp. 7800211, Aug. 2013.

[10] L. Zeng, D. O’Brien, H. Minh, G. Faulkner, K. Lee, D. Jung, Y. J. Oh, and E. T. Won, "High data rate multiple input multiple output (MIMO) optical wireless communications using white LED lighting," IEEE Journal on Selected Areas in Communications, vol. 27, no. 9, pp. 1654-1662, Dec. 2009.

[11] T. Fath and H. Haas, "Performance comparison of MIMO techniques for optical wireless communications in indoor environments," IEEE Transactions on Communications, vol. 61, no. 2, pp. 733-742, Feb. 2013.

[12] D. Tsonev, H. Chun, S. Rajbhandari, J. J. D. McKendry, S. Videv, E. Gu, M. Haji, S. Watson, A. E. Kelly, G. Faulkner, M. D. Dawson, H. Haas, and
D. O'Brien, “A 3-Gb/s single-LED OFDM-based wireless VLC link using a Gallium nitride $\mu$ LED," IEEE Photonics Technology Letters, vol. 26, no. 7, pp. 637-640, Apr. 2014.

[13] Z. Wang, Q. Wang, S. Chen, and L. Hanzo, "An adaptive scaling and biasing scheme for OFDM-based visible light communication systems," Optics Express, vol. 22, no. 10, pp. 12707-12715, May 2014.

[14] Q. Wang, Z. Wang, and L. Dai, "Iterative receiver for hybrid asymmetrically clipped optical OFDM," Journal of Lightwave Technology, vol. 32, no. 22, pp. 3869-3875, Nov. 2014.

[15] Q. Wang, C. Qian, X. Guo, Z. Wang, D. G. Cunningham, and I. H. White, "Layered ACO-OFDM for intensity-modulated directdetection optical wireless transmission," Optics Express, vol. 23, no. 9, pp. 12382-12393, May 2015.

[16] Q. Wang, Z. Wang, and L. Dai, "Asymmetrical hybrid optical OFDM for visible light communications with dimming control," IEEE Photonics Technology Letters, vol. 27, no. 9, pp. 974-977, May 2015.

[17] G. L. Stuber, J. R. Barry, S. W. Mclaughlin, Y. Li, M. A. Ingram, and T. G. Pratt, "Broadband MIMO-OFDM wireless communications," Proceedings of the IEEE, vol. 92, no. 2, pp. 271-294, Feb. 2004.

[18] H. Yang, "A road to future broadband wireless access: MIMO-OFDMbased air interface," IEEE Commununications Magazine, vol. 43, no. 1, pp. 53-60, Jan. 2005.

[19] A. H. Azhar, T. Tran, and D. O'Brien, "A gigabit/s indoor wireless transmission using MIMO-OFDM visible-light communications," IEEE Photonics Technology Letters, vol. 25, no. 2, pp. 171-174, Jan. 2013.

[20] A. C. Boucouvalas, K. Yiannopoulos and Z. Ghassemlooy, "100 Gbit/s optical wireless communication system link throughput," Electronics Letters, vol. 50, no. 17, pp. 1220-1222, Aug. 2014.

[21] D. Tsonev, S. Videv, and H. Haas, "Towards a $100 \mathrm{~Gb} / \mathrm{s}$ visible light wireless access network," Optics Express, vol. 23, no. 2, pp. 1627-1637, Jan. 2015.

[22] Q. H. Spencer, A. L. Swindlehurst, and M. Haardt, "Zero-forcing methods for downlink spatial multiplexing in multiuser MIMO channels," IEEE Transactions on Signal Processing, vol. 52, no. 2, pp. 461-471, Feb. 2004.

[23] Q. H. Spencer, C. B. Peel, A. L. Swindlehurs, and M. Haardt, "An introduction to the multi-user MIMO downlink," IEEE Communications Magazine, vol. 42, no. 10, pp. 60-67, Oct. 2004.

[24] S. D. Dissanayake and J. Armstrong, "Comparison of ACO-OFDM, DCO-OFDM and ADO-OFDM in IM/DD systems," Journal of Lightwave Technology, vol. 31, no. 7, pp. 1063-1072, Apr. 2013. 